\title{
ON A PROBLEM OF BAAYEN AND KRUYSWIJK
}

\author{
by D. A. BURGESS \\ (Received 13th December 1967)
}

1. We shall call a finite semigroup $S$ arithmetical if there exists a positive integer $N$ and a monomorphism $\mu$ of $S$ into the multiplicative semigroup $R_{N}$ of the ring of residue classes of the integers modulo $N$. In 1965 P. C. Baayen and D. Kruyswijk [1] posed the problem ' Is every finite commutative semigroup arithmetical?' The purpose of this paper is to answer this question.

In section $2 \mathrm{I}$ obtain a necessary and sufficient condition for a finite semigroup to be arithmetical. In section 3 I use this criterion to demonstrate that there are finite commutative semigroups which are not arithmetical. In section 4 I use the criterion to prove that certain special classes of commutative semigroups are arithmetical. Finally in section $5 \mathrm{I}$ give the weaker theorem that every finite commutative semigroup is a homomorphic image of an arithmetical semigroup.

2. Let $S$ be a finite commutative semigroup. Let $C$ denote the set of roots of unity (in the field of complex numbers) and let $x$ be an indeterminate. We shall define $\chi$ to be a character of $S$ if there is a positive integer $m$ such that $\chi$ is a homomorphism of $S$ into the semigroup of elements

$$
\omega x^{\alpha}\left(\bmod x^{m}\right)
$$

under multiplication, where $\omega$ is in $C$ and $\alpha$ is a non-negative integer. [This is not the usual definition of a semigroup character (see A. H. Clifford and G. B. Preston [2]), but it is a convenient notation for our investigation.] These mappings become representations if we interpret $x$ as a matrix satisfying

$$
x^{m}=0, \quad x^{m-1} \neq 0 \text {. }
$$

If $S$ is a group then the Abelian group characters are characters in our sense with

$$
m=1 \text {. }
$$

Our principal result is:

Theorem 1. A finite commutative semigroup $S$ is arithmetical if and only if for each pair of distinct elements $a, b$ of $S$ there is a character $\chi=\chi_{a, b}$ of $S$ for which

$$
\chi(a) \neq \chi(b) .
$$

Proof. Suppose that $S$ is arithmetical. Thus $S$ can be embedded isomorphically in some $R_{N}$. We show that for each pair $a, b$ of distinct elements of $R_{N}$ there is a $\chi$ of $R_{N}$ satisfying (1). 
Let the canonical factorisation of $N$ be

$$
N=\prod_{i=1}^{r} p_{i}^{\alpha(i)}
$$

Then $R_{N}$ can be represented as the direct sum

$$
R_{N}=\sum_{i=1}^{r} R_{p_{i}^{\alpha}(i)}
$$

For, by the Chinese remainder theorem, we may choose $k_{1}, \ldots, k_{r}$ such that

$$
k_{i} \equiv \begin{cases}1\left(\bmod p_{i}^{\alpha(i)}\right) & (i=1, \ldots, r), \\ 0\left(\bmod p_{j}^{\alpha(j)}\right) & (j \neq i),\end{cases}
$$

and then the representation

$$
x \equiv \sum_{i=1}^{r} k_{i} x_{i}(\bmod N)
$$

gives the required isomorphism, where each $x_{i}$ runs through the residue classes modulo $p_{i}^{\alpha(i)}$. Since $a$ and $b$ are distinct there is an $i$ for which $a_{i}$ and $b_{i}$ are distinct. Thus, by applying first the projection homomorphism of $R_{N}$ onto $\boldsymbol{R}_{\boldsymbol{p}_{l}^{\alpha(i)}}$, it suffices to prove the result in the restricted case when $N=p^{\alpha}$ is a power of a prime $p$.

Now if

$$
\left(a, p^{\alpha}\right) \neq\left(b, p^{\alpha}\right)
$$

where $(u, v)$ denotes the highest common factor of $u$ and $v$, then the character $\chi$ defined by

$$
\chi(z)=x^{5}\left(\bmod x^{x}\right),
$$

where $\left(z, p^{\alpha}\right)=p^{\zeta}$, satisfies (1). If alternatively

$$
\left(a, p^{\alpha}\right)=\left(b, p^{\alpha}\right)=p^{\beta} \text { say, }
$$

let $\psi$ be an Abelian group character of the multiplicative group of residua classes modulo $p^{\alpha-\beta}$ that are relatively prime to $p$ for which

$$
\psi\left(a / p^{\beta}\right) \neq \psi\left(b / p^{\beta}\right) .
$$

In this case we define the character $\chi$ by

$$
\chi(z)=\psi\left(z / p^{\zeta}\right) x^{\zeta}\left(\bmod x^{\beta+1}\right)
$$

and again (1) is satisfied.

Next we prove the converse. Let $S$ be a semigroup and $a$ and $b$ be two distinct elements of $S$. Let $\chi=\chi_{a, b}$ be a character of $S$ such that

$$
\chi(a) \neq \equiv(b)\left(\bmod x^{m}=x^{m(a, b)}\right) \text {. }
$$

Let $d=d_{a, b}$ be the least common multiple of the orders of the coefficients of the non-zero monomials which belong to $\operatorname{Im} \chi$. Choose an odd prime $p=p_{a, b}$ for which

$$
p \equiv 1 \quad(\bmod d)
$$


Let $g$ be a primitive $d$ th root of unity modulo $p^{m}$, and so also modulo $p$, and let $\eta$ be a primitive $d$ th root of unity in $C$. Then the map $\tau=\tau_{a, b}$ given by

$$
\tau\left(\eta^{\lambda} x^{\zeta}\right)=g^{\lambda} p^{\zeta}\left(\bmod p^{m}\right)
$$

is a monomorphism of $\operatorname{Im} \chi$ into $R_{p^{m}}=R_{a, b}$ say, and so the homomorphism $\tau \chi$ of $S$ into $R_{a, b}$ has the property

$$
\tau \chi(a) \neq \tau \chi(b)
$$

By Dirichlet's theorem we may choose all the odd primes $p_{a, b}$ (required only to satisfy (2)) to be distinct. Thus there is a monomorphism

where

$$
\mu: S \rightarrow \sum_{a, b} R_{a, b} \simeq R_{N},
$$

$$
N=\prod_{a, b} p_{a, b}^{m(a, b)},
$$

the component of $\mu$ in $R_{a, b}$ being $\tau_{a, b} \chi_{a, b}$.

3. Theorem 2. There exists a non-arithmetical finite commutative semigroup.

Proof. We show that the semigroup $S$ given by the multiplication table

\begin{tabular}{l|llll} 
& $e$ & $a$ & $b$ & $c$ \\
$e$ & $e$ & $e$ & $e$ & $e$ \\
$b$ & $e$ & $e$ & $c$ & $e$ \\
$c$ & $e$ & $c$ & $e$ & $e$ \\
$e$ & $e$ & $e$ & $e$
\end{tabular}

is not arithmetical. It suffices to show that there is no character $\chi$ of $S$ with

$$
\chi(e) \neq \chi(c) \text {. }
$$

Since $e$ is an idempotent and $e c=e$ we must have

Next we have

$$
\chi(e) \equiv 0 \quad\left(\bmod x^{m}\right)
$$

Thus

$$
\chi(a)^{2} \equiv \chi(b)^{2} \equiv \chi(e) \equiv 0 \quad\left(\bmod x^{m}\right) \text {. }
$$

which implies that

$$
\chi(c)^{2} \equiv \chi(a)^{2} \chi(b)^{2} \equiv 0 \quad\left(\bmod x^{2 m}\right)
$$

$$
\chi(c) \equiv 0 \equiv \chi(e) \quad\left(\bmod x^{m}\right)
$$

and so (3) is not satisfied.

4. Theorem 3. A finite direct sum of arithmetical semigroups is arithmetical.

Proof. If $a, b$ are two distinct elements of the direct sum $S$ we let $\tau$ be the projection homomorphisms of $S$ onto some component $T$ in which $\tau(a) \neq \tau(b)$. Then since $T$ is arithmetical there is a character $\chi$ of $T$ with

$$
\chi \tau(a) \neq \chi \tau(b)
$$

so that $\chi \tau$ is the required character of $S$. 
Theorem 4. If the finite commutative semigroup $S$ can be partitioned into a set of disjoint groups then $S$ is arithmetical.

Proof. Let

$$
S=\bigcup_{i=1}^{n} S_{i}
$$

where each $S_{i}$ is a group and has a unique idempotent $e_{i}$ (the identity).

Now we note that the relation $>$ defined by

$$
S_{i}>S_{j} \text { if and only if } e_{i} e_{j}=e_{i}
$$

is a partial ordering of the $S_{i}$. Also to each pair of integers $i, j$ there corresponds a unique integer $k$ such that

$$
S_{i} S_{j} \subset S_{k}
$$

since each element of $S_{i} S_{j}$ contains among its powers the idempotent $e_{i} e_{j}=e_{k}$ say, and so belongs to $S_{k}$. Further we see that if (4) holds then $S_{i}<S_{k}$ for we have

$$
e_{i} e_{k}=e_{i} e_{i} e_{j}=e_{i} e_{j}=e_{k} .
$$

Next we consider a pair of distinct elements $a, b$ of $S$. Suppose that

$$
a \in S_{i}, \quad b \in S_{j} \text {. }
$$

If $i \neq j$ then at most one of $S_{i}<S_{j}$ and $S_{j}<S_{i}$ can hold. We may assume without loss of generality that $S_{j}<S_{i}$ is false. We define the character $\chi$ by

where if $z \in S_{l}$ we have

$$
\chi(z)=x^{5} \quad(\bmod x)
$$

$$
\zeta=\left\{\begin{array}{l}
0 \text { if } S_{l}<S_{i} \\
1 \text { otherwise. }
\end{array}\right.
$$

This $\chi$ satisfies (1). On the other hand if $i=j$ there is a character $\psi$ of $S_{i}$ with Then we define $\chi$ by

$$
\psi(a) \neq \psi(b)
$$

and again $\chi$ satisfies (1).

$$
\chi(z)= \begin{cases}\psi\left(z e_{i}\right) & \text { if } S_{l}<S_{i} \\ 0 & \text { otherwise }\end{cases}
$$

5. Theorem 5. Any finite commutative semigroup $S$ is a homomorphic image of an arithmetical semigroup.

Proof. Let $z_{1}, \ldots, z_{n}$ be the elements of $S$. We may consider $S$ as a commutative semigroup with generators $z_{1}, \ldots, z_{n}$ and a certain set $R$ of relations. For each $z_{i}$ there is a positive integer $n_{i}$ such that $z_{i}^{n_{i}}$ is an idempotent. Write

Then for each $z_{i}$

$$
M=\prod_{i=1}^{n} n_{i}
$$

$$
z_{i}^{2 M}=z_{i}^{M}
$$


is a relation in $R$. Define $T$ to be the commutative semigroup with generators $z_{1}, \ldots, z_{n}$ and relations (5) for $i=1, \ldots, n$. Thus $S$ is a homomorphic image of $T$. It suffices to show $T$ is arithmetical.

By Theorem 3 it is sufficient to show that the semigroup $U$ on one generator $z$ with the relation

$$
z^{2 M}=z^{M}
$$

is arithmetical, for $T$ is a direct sum of $n$ copies of $U$. Choose two elements $z^{i}, z^{j}$ of $U$. If at least one of $i, j$ is less than $M$ then the character $\chi$ defined by

has the property

$$
\begin{gathered}
\chi\left(z^{k}\right)=x^{k} \quad\left(\bmod x^{m}\right) \\
\chi\left(z^{i}\right) \neq \chi\left(z^{j}\right) .
\end{gathered}
$$

On the other hand if both $i$ and $j$ belong to the closed interval $[M, 2 M-1]$ then the character $\chi$ given by

$$
\chi\left(z^{k}\right)=e^{2 \pi i k / M}(\bmod x)
$$

satisfies (6), and our proof is complete.

\section{REFERENCES}

(1) P. C. BAaYen and D. KRuYswijk, A note on the multiplicative semigroup of the residue classes modulo $n$, Math. Centrum Amsterdam Afd. Zuivere Wisk. $\mathrm{ZW}$ $1965-007$.

(2) A. H. Clifford and G. B. Preston, The Algebraic Theory of Semigroups, vol.1, Math. Surveys of the American Math. Soc. 7 (Providence, R. I., 1961).

THE UNIVERSITY

NotTINGHAM 